\title{
RESISTÊNCIA DE GENÓTIPOS DE FEIJÃO-CAUPI AO ATAQUE DE CALLOSOBRUCHUS MACULATUS (FABR., 1775) (COLEOPTERA: CHRYSOMELIDAE: BRUCHINAE)
}

\author{
A.F. de Melo ${ }^{1 *}$, L.S. Fontes ${ }^{2}$, D.R.S. Barbosa ${ }^{1 *}$, \\ A.A.R. Araújo ${ }^{1 *}$, E.P.S. Sousa ${ }^{1 *}$, L.L.L. Soares ${ }^{1 *}$, P.R.R. Silva ${ }^{3}$ \\ ${ }^{1}$ Universidade Federal do Piauí, Campus da Socopo, CEP 64049-550, Teresina, PI, Brasil. E-mail: alynefmelo@ \\ yahoo.com.br
}

\section{RESUMO}

\begin{abstract}
O objetivo deste trabalho foi avaliar a resistência de quatro genótipos de feijão-caupi (Vigna unguiculata) ao caruncho Callosobruchus maculatus (Fabr., 1775). Os genótipos utilizados foram BR 17-Gurguéia, BRS Rouxinol, TE96-290-12G e BRS Guariba. Foram realizados testes com e sem chance de escolha, em delineamento experimental inteiramente casualizado, totalizando 4 tratamentos cada um com 5 repetições, avaliando-se número de ovos, número de insetos emergidos, viabilidade de ovos (\%) e taxa instantânea de crescimento populacional. Nos testes com e sem chance de escolha, o genótipo TE96-290-12G mostrou-se como o mais resistente. BRS Rouxinol foi o genótipo mais suscetível.
\end{abstract}

PALAVRAS-CHAVE: Vigna unguiculata, caruncho, grãos armazenados, controle alternativo.

\author{
ABSTRACT
}

\begin{abstract}
RESISTANCE OF GENOTYPES OF COWPEA TO THE ATTACK OF CALLOSOBRUCHUS MACULATUS (FABR., 1775) (COLEOPTERA: CHRYSOMELIDAE: BRUCHINAE). The objective of this study was to evaluate the resistance of 4 genotypes of cowpea (Vigna unguiculata) to the bean weevil Callosobruchus maculatus (Fabr., 1775). The genotypes evaluated were BR 17-Gurguéia, BRS Rouxinol, TE96-290-12G and BRS Guariba. Tests were conducted with and without possibility of choice, in a completely randomized design, totaling 4 treatments each with 5 replicates, evaluating the number of eggs, number of emerged insects, egg viability (\%) and instantaneous rate of population growth. In the test with possibility of choice the genotype TE96-290-12G was revealed as the most resistant. BRS Rouxinol was the most susceptible genotype.
\end{abstract}

KEY WORDS: Vigna unguiculata, bean weevil, stored grains, alternative control.

O feijão-caupi ou feijão-de-corda [Vigna unguiculata (L.) Walp.] é um componente da dieta alimentar de povos em países subdesenvolvidos. Sua importância está no alto conteúdo de proteína nas sementes (AKANDE, 2007). No Brasil, o caupi é cultivado, basicamente, em regime de subsistência, nas regiões Norte e Nordeste, principalmente por sua adaptação às condições edafoclimáticas (ZILLI et al., 2004).

O feijão-caupi tem significativa importância socioeconômica como suprimento alimentar, na fixação de mão-de-obra no campo e como componente da produção agrícola, especialmente nas regiões Norte e Nordeste (BezErRaet al., 2008). Devido às condições de adaptabilidade e do hábito alimentar da população, o feijão-caupi é cultivado predominantemente nestas regiões, alcançando quase a totalidade das áreas plantadas com feijão no Amazonas, Rio Grande do Norte, Ceará, Piauí e Maranhão (SAntos; Araújo, 2000).

Dentre as pragas que atacam grãos de feijão durante o armazenamento, destaca-se o gorgulhodo-feijão, Callosobruchus maculatus (Fabr., 1775) (Coleoptera: Chrysomelidae: Bruchinae), por reduzir a qualidade e o valor comercial do produto (SousA et al., 2005). A redução da qualidade dos grãos durante o armazenamento está associada, principalmente, ao grau de infestação dos grãos e às condições

${ }^{2}$ Universidade Federal do Piauí, Departamento de Biologia, Campus Ministro "Petrônio Portela", Teresina, PI, Brasil. ${ }^{3}$ Universidade Federal do Piauí, Departamento de Fitotecnia, Laboratório de Fitossanidade, Campus da Socopo, Teresina, PI, Brasil.

*Programa de Pós-Graduação em Agronomia/Produção Vegetal. 
ambientais em queencontra a massa de grãos (FARONI; SOUSA, 2006).

O caruncho, C. maculatus, é considerado a praga mais importante do caupi armazenado em regiões tropicais e subtropicais (Pereira et al., 2008). Tem seus danos decorrentes da penetração e alimentação das larvas no interior das sementes, provocando perda de peso, redução do poder germinativo, do valor nutritivo das sementes e grãos, e do grau de higiene do produto, pela presença de excrementos, ovos e insetos (ALMEIDA et al., 2005).

Seu controle, assim como dos demais insetospraga associados aos grãos armazenados, tem sido realizado em larga escala, por meio de produtos químicosfumigantes. Relatos sobre desenvolvimento deresistência a tratamentos químicos em várias espécies de insetos-praga são cada vez mais constantes (Martinazzoet al., 2000). Ouso irrestrito de produtos químicos também pode ocasionar problemas de contaminação ambiental devido a efeitos residuais e intoxicação de humanos e animais.

O uso de cultivares que possuam algum tipo de resistência genética ao inseto constitui um método de controle promissor para o controle de C. maculatus na região Nordeste. Nessa linha, várias pesquisas têm sido conduzidas, procurando-se estudar genótipos de caupi que possam apresentar resistência a $C$. maculatus (Mota et al., 2002). Segundo ApplebY; CRedland (2004), o desenvolvimento e a liberação de variedades resistentes de caupi representam uma alternativa atrativa aos métodos químicos convencionais para o controle de $C$. maculatus.

Neste contexto, objetivou-se avaliar a resistência de genótipos de feijão-caupi ao ataque C. maculatus, visando a fornecer um método de controle alternativo ao uso de produtos químicos.

O trabalho foi desenvolvido no Laboratório de Entomologia, Departamento de Biologia, Centro de Ciências da Natureza da Universidade Federal do Piauí. Para a instalação do experimento foram utilizados insetos da espécie C. maculatus, provenientes da criação estoque mantida no próprio laboratório, em vidro fechado de $5 \mathrm{~L}$ com tampas revestidas com lenço de papel, tipo "Yes", a fim de permitir as trocas gasosas e evitar a penetração de ácaros ou inimigos, sob temperatura de $30 \pm 2^{\circ} \mathrm{C}$ e $70 \pm 5 \%$ de umidade relativa, em sala climatizada.

Foram utilizadosquatro genótipos de feijão-caupi provenientes da Embrapa Meio-Norte: BRSGuariba, BR 17-Gurguéia, BRS Rouxinol e TE96-290-12G. Os grãos foram acondicionados em sacos plásticos em congelador à temperatura de $-5^{\circ} \mathrm{C}$ para eliminação de eventuais infestações latentes. Antes da instalação dos experimentos, os grãos foram retirados do congelador, colocados em recipientes plásticos cobertos com tecido fino para entrarem em equilíbrio higroscópico.
A análise da resistência foi feita com a realização de dois testes: come sem chance de escolha aos genótipos avaliados. Para o teste com chance de escolha foram colocados 30 insetos adultos do caruncho $C$. maculatus (idade de 0 a 24 horas sem determinação do sexo) em uma arena plástica contendo cinco compartimentos interligados: um central para os insetos e os demais contendo 50 grãos de cada genótipo. Após 24 horas da infestação, as interligações entre os compartimentos foram fechadas com algodão e após oito dias foi feita a contagem de ovos por genótipo.

$\mathrm{O}$ teste sem chance de escolha foi realizado em placas de Petri medindo $2 \mathrm{~cm}$ de altura por $15 \mathrm{~cm}$ de diâmetro, cada uma com 50 grãos de cada genótipo de feijão-caupi infestados por 10 insetos adultos de C. maculatus com idade de 0 a 24 horas, sem determinação do sexo. A contagem de ovos por genótipo foi feita oito dias após a infestação.

Na avaliação do efeito dos genótipos foram utilizados os parâmetros número de ovos, número de insetos emergidos, viabilidade de ovos (\%) e taxa instantânea de crescimento populacional (ri), para o cálculo desta utilizou-se a equação (WALTHALL; STARK, 1997): $\mathrm{ri}=\left[\ln \left(\mathrm{N}_{\mathrm{f}} / \mathrm{N}_{0}\right) / \Delta \mathrm{T}\right]$, onde $\mathrm{N}_{\mathrm{f}}=$ Número final de insetos; $\mathrm{N}_{0}=$ Número inicial de insetos; e $\Delta \mathrm{T}=$ Variação de tempo (número de dias em que o ensaio foi executado).

O delineamento experimental adotado nos dois testes foi inteiramente casualizado, com quatro tratamentos e cinco repetições. Os dados foram submetidos à análise de variância pelo teste $\mathrm{F}$ a 5\% de probabilidade e as médias comparadas pelo teste de Tukey a $5 \%$ de probabilidade. Na análise da taxa instantânea de crescimento populacional (ri) de $C$. maculatus em genótipos de feijão-caupi em teste com chance de escolha os dados originais foram transformados para $(x+0,5)^{1 / 2}$.

A oviposição por genótiponão variou significativamentenotestecom chance deescolha(Tabela1). PEssOA et al. (1993) descreveram o genótipo IPA 206 como um dos mais preferidos para oviposição de C. maculatus, dentre 10 genótipos por eles estudados. No presente trabalho, ogenótipo TE96-290-12Gnotestecomchance de escolha apresentou a menor emergência entre todos os genótipos, mas não diferiu estatisticamente do genótipo BR17-Gurguéia. Sendo assim, TE96-290-12G foi o genótipo mais resistente ao ataque de C. maculatus em relação ao parâmetro avaliado.

Com base nos resultados obtidos no teste sem chance de escolha, ogenótipo TE96-290-12G apresentou uma baixa emergência, indicando que apresenta resistência do tipo antibiose (Tabela 1). ARAúJo; WatT (1988) consideraram que a resistência do tipo antibiose é caracterizada, sobretudo, pelo alongamento do período de ovo a adulto e pela redução do número de adultos de $C$. maculatus emergidos em posturas efetuadas nas sementes e vagens de caupi. 
Tabela 1 - Oviposição e emergência de C. maculatus, em genótipos de caupi, em teste com e sem chance de escolha. Teresina, PI, 2010.

\begin{tabular}{|c|c|c|c|c|}
\hline \multirow{2}{*}{ Genótipos } & \multicolumn{2}{|c|}{ Teste com chance de escolha } & \multicolumn{2}{|c|}{ Teste sem chance de escolha } \\
\hline & № de ovos ${ }^{1}$ & № de adultos emergidos ${ }^{1}$ & № de ovos ${ }^{1}$ & № de adultos emergidos ${ }^{1}$ \\
\hline BRS Guariba & $450,00 \mathrm{a}$ & $296,25 a$ & $361,00 c$ & $329,00 \mathrm{~b}$ \\
\hline BR 17-Gurguéia & $526,25 a$ & $231,25 \mathrm{ab}$ & $553,00 \mathrm{~b}$ & $273,00 \mathrm{~b}$ \\
\hline BRS Rouxinol & $676,25 a$ & $350,00 \mathrm{a}$ & $712,00 \mathrm{a}$ & $459,00 \mathrm{a}$ \\
\hline TE96-290-12G & $418,75 a$ & $160,00 \mathrm{~b}$ & $538,00 \mathrm{ab}$ & $252,00 \mathrm{~b}$ \\
\hline C.V. $(\%)$ & 27,05 & 23,05 & 17,36 & 20,38 \\
\hline
\end{tabular}

${ }^{1}$ Médias seguidas da mesma letra na coluna não diferem significativamente a $5 \%$ de probabilidade, pelo teste de Tukey.

Tabela 2 - Viabilidade de ovos e taxa instantânea de crescimento populacional (ri) de C. maculatus, em genótipos de caupi, em teste com e sem chance de escolha. Teresina, PI, 2010.

\begin{tabular}{|c|c|c|c|c|}
\hline \multirow[b]{2}{*}{ Genótipos } & \multicolumn{2}{|c|}{ Teste com chance de escolha } & \multicolumn{2}{|c|}{ Teste sem chance de escolha } \\
\hline & $\begin{array}{l}\text { Viabilidade } \\
\text { de ovos }(\%)^{1}\end{array}$ & $\begin{array}{c}\text { Taxa instantânea de } \\
\text { crescimento populacional (ri) }{ }^{2}\end{array}$ & $\begin{array}{l}\text { Viabilidade } \\
\text { de ovos }(\%)^{1}\end{array}$ & $\begin{array}{c}\text { Taxa instantânea de } \\
\text { crescimento populacional (ri) }\end{array}$ \\
\hline BRS Guariba & $66,54 \mathrm{a}$ & $0,022 \mathrm{ab}$ & $89,16 a$ & $0,060 \mathrm{ab}$ \\
\hline BR 17-Gurguéia & $45,90 \mathrm{a}$ & $0,012 \mathrm{ab}$ & $52,74 \mathrm{bc}$ & $0,058 \mathrm{ab}$ \\
\hline BRS Rouxinol & $52,19 a$ & $0,027 \mathrm{a}$ & $64,70 \mathrm{~b}$ & $0,072 \mathrm{a}$ \\
\hline TE96-290-126 & $42,19 a$ & $0,000 \mathrm{~b}$ & $45,06 \mathrm{c}$ & $0,052 b$ \\
\hline C.V. $(\%)$ & 26,65 & 1,14 & 12,67 & 16,73 \\
\hline
\end{tabular}

${ }^{1}$ Médias seguidas da mesma letra na coluna não diferem significativamente a $5 \%$ de probabilidade, pelo teste de Tukey. ${ }^{2}$ Dados originais; para análise foram transformados em $(x+0,5)^{1 / 2}$, médias seguidas da mesma letra na coluna não diferem significativamente a $5 \%$ de probabilidade, pelo teste de Tukey.

No teste sem chance de escolha, em relação ao número de ovos, o genótipo BRS Guariba apresentou a menor oviposição diferindo estatisticamente dos demais.

Lima et al. (2001) destacaram o genótipo CNCX 40912F como suscetível a C. maculatus por apresentar maior viabilidade de ovos, maior número de insetos emergidos e menor período de ovo a adulto. No presente trabalho, em teste sem chance de escolha, o genótipo BRS Rouxinol foi o mais ovipositado e apresentou maior número de insetos emergidos mostrando-se suscetível ao ataque do caruncho

A viabilidade média de ovos não apresentou variação significativa entre os genótipos no teste com chance de escolha, contudo, no teste sem chance de escolha observou-se diferença significativa entre os genótipos (Tabela 2). Entre os genótipos analisados em teste sem chance de escolha, o genótipo TE96290-12G apresentou uma baixa viabilidade de ovos, sendo significativamente menor que os demais, já o genótipo BRS Guariba apresentou a maior viabilidade de ovos. Os genótipos BRS Rouxinol e BR 17-Gurguéia apresentaram valores intermediários na viabilidade de ovos. Lima et al. (2001) estudando a resistência de caupi a C. maculatus observaram uma viabilidade de ovos de $82,8 \%$ para o genótipo BR 17-Gurguéia, no presente trabalho observou-se uma viabilidade de 45,90 e $52,74 \%$, nos testes com e sem chance de escolha, respectivamente.
Ribeiro-Costa et al. (2007), buscando verificar o desenvolvimento de Z. subfasciatus em genótipos de $P$. vulgaris sem e com arcelina, demonstraram que genótipos como IAPAR 44 sem arcelina, ARC1 e ARC2 contendo esta proteína, apresentaram maior resistência, pois obtiveram menor preferência para oviposição, e baixo percentual de ovos viáveis. No presente trabalho, a baixa viabilidade de ovos no genótipo TE96-290-12G, em teste sem chance de escolha, deve-se provavelmente à presença de substâncias inibidoras da alimentação de C. maculatus, como exemplo a presença de proteína arcelina nos grãos deste genótipo. A presença de substâncias inibidoras de alimentação em carunchos é relatada na literatura, a exemplo da arcelina que confere resistência a $Z$. subfasciatus em feijoeiro (ORIANI; LARA, 2000) e inibidores de tripsina responsáveis pela antibiose em alguns genótipos de feijão-caupi (GATEHOUSE et al., 1989).

A taxa instantânea de crescimento populacional diferiu significativamente entre os genótipos no teste com e sem chance de escolha, em ambos os testes o genótipo TE96-290-12G foi significativamente diferente do genótipo BRS Rouxinol que apresentou uma alta taxa de crescimento populacional. Nos dois testes os genótipos BRS Guariba e BR 17-Gurguéia apresentaram valores intermediários.

Segundo LARA (1991), nem sempre os genótipos mais ovipositados são os mais suscetíveis, porque 
poderão existir outros fatores que impeçam o desenvolvimento larval do inseto e, dessa forma, um genótipo muito ovipositado pode ainda revelar-se resistente. BARRETO; QUINDERÉ (2000) observaram que as variáveis número deovos, número deinsetos emergidos e número de sementes de caupi danificadas por C. maculatus mostraram-se positivas e significativamente correlacionadas entre si. No presente trabalho, a taxa instantânea de crescimento populacional, a oviposição e a emergência por genótipo apresentaram correlação positiva entre si, com isso, pode-se afirmar que nos dois testes os genótipos mais ovipositados apresentam também maior emergência por genótipo e maior taxa instantânea decrescimento populacional, mostrando-se menos resistentes.

Levando-se em consideração os resultados expostos, foi possível verificar que o genótipo TE96290-12G apresentou resistência do tipo antibiose e o genótipo BRS Rouxinol foi o mais suscetível ao ataque de C. maculatus

\section{REFERÊNCIAS}

AKANDE, S.R. Genotype by environment interaction for cowpea seed yield and disease reactions in the forest and derived savanna agro-ecologies of southwest Nigeria. American-Eurasian Journal of Agricultural \& Environmental Science, v.2, n.2, p.163-168, 2007.

ALMEIDA, F. de A.C.; ALMEIDA, S.A. de; SANTOS, N.R. dos; GOMES, J.P.; ARAÚJO, M.E.R. Efeitos de extratos alcoólicos de plantas sobre o caruncho do feijão vigna (Callosobruchus maculatus). Revista Brasileira de Engenharia Agrícola e Ambiental, v.9, n.4, p.585-590, 2005.

APPLEBY, J.H.; CREDLAND, P.F. Environmental conditions affect the response of West African Callosobruchus maculatus (Coleoptera: Bruchidae) populations to susceptible and resistant cowpeas. Journal of Stored Products Research, v.40, n.3, p.269-287, 2004.

ARAÚJO, J.P.P.; WATT, E.E. O caupi no Brasil. Brasília: EMBRAPA-CNPAF, 1988. 722p.

BARRETO, P.D.; QUINDERÉ, A.W. Resistência de genótipos de caupi ao caruncho. Pesquisa Agropecuária Brasileira, v.35, n.4, p.779-785, 2000.

BEZERRA, A.A. de C.; TÁVORA, F.J.A.F.; FREIRE FILHO, F.R.; RIBEIRO, V.Q. Morfologia e produção de grãos em linhagens modernas de feijao-caupi submetidas a diferentes densidades populacionais. Revista de Biologia e Ciências da Terra, v.8, n.1, p.85-93, 2008.

FARONI, L.R.A.; SOUSA, A.H. Aspectos biológicos e taxonômicos dos principais insetos-praga de produtos armazenados. In: ALMEIDA, F.A.C.; DUARTE, M.E.M.; MATA, M.E.R.M.C. (Ed.). Tecnologia de armazenagem em sementes. Campina Grande: UFCG, 2006. p.371-402.
GATEHOUSE, A.M.R.; GATEHOUSE, J.A; DOBIE, P.; KILMINSTER, A.M.; BOULTIER, D. Biochemical basis of insect resistance in Vigna unguiculata. Journal of the Science of Food and Agriculture, v.30, n.10, p.948-958, 1989.

LARA, F.M. Princípios de resistência de plantas a insetos. 2.ed. São Paulo: Ícone, 1991. 336p.

LIMA, M.P.L.; OLIVEIRA, J.O.; BARROS, R.; TORRES, J.B.; GONÇALVES, M.E.C. Estabilidade da resistência de genótipos de caupi a Callosobruchus maculatus (Fabr.) em gerações sucessivas. Scientia Agricola, v.59, n.2, p.275-280, 2001.

MARTINAZZO, A.P.; FARONI, L.R.D.; BERBERT, P.A.; REIS, F.P. Utilização da fosfina em combinação com o dióxido de carbono no controle do Rhyzopertha dominica (f.). Pesquisa Agropecuária Brasileira, v.35, n.6, p.10631069, 2000.

MOTA, A.C.; FERNANDES, K.V.S.; SALES, M.P.; FLORES, V.M.Q.; XAVIER FILHO, E. Cowpea vicilins: fraction of urea denatured sub-units and effects on Callosobruchus maculatus F. (Coleoptera: Bruchidae) development. Brazilian Archives of Biology and Technology, v.45, n.1, p.1-5, 2002.

ORIANI, M.A. de G.; LARA, F.M. Antibiosis effects of wild bean lines containing arcelin on Bemisia tabaci (Genn.) biotype B (Homoptera: Aleyrodidae). Anais da Sociedade Entomológica do Brasil, v.29, n.3, p.573-582, 2000.

PEREIRA, A.C.R.L.; OLIVEIRA, J.V. de; GONDIM JUNIOR, M.G.C.; CÂMARA, C.A.G. da Atividade inseticida de óleos essenciais e fixos sobre Callosobruchus maculatus (Fabr., 1775) (Coleoptera: Bruchidae) em grãos de caupi [Vigna unguiculata (L.) Walp.]. Ciência e Agrotecnologia, v.32, n.3, p.717-724, 2008.

PESSOA, G.P.; BARROS, R.; OLIVEIRA, J.V. Avaliação da resistência de cultivares de caupi (Vigna unguiculata (L.) Walp.) a Callosobruchus maculatus em confinamento em laboratório. Anais da Sociedade Entomológica do Brasil, v.22, n.2, p.259-266, 1993.

RIBEIRO-COSTA, C.S.; PEREIRA, P.R.V.S.; ZUKOVSKI, L. Desenvolvimento de Zabrotes subfasciatus (Boh.) (Coleoptera: Chrysomelidae, Bruchidae) em genótipos de Phaseolus vulgaris L. (Fabaceae) cultivados no Estado do Paraná e contendo arcelina. Neotropical Entomology, v.36, n.4, p.560-564, 2007.

SANTOS, C.A.F.; ARAÚJO, F.P. Produtividade e morfologia de genótipos de caupi em diferentes densidades populacionais nos sistemas irrigado e de sequeiro. Pesquisa Agropecuária Brasileira, v.35, n.10, p.1977-1984, 2000.

SOUSA, A.H.; MARACAJÁ, P.B.; SILVA, R.M.A. da; MOURA. A.M.N; ANDRADE. W.G de. Bioactivity of 
vegetal powders against Callosobruchus maculatus (Coleoptera: Bruchidae) in caupi bean and seed physiological analysis. Revista de Biologia e Ciências da Terra, v.5, n.2, p.1519-5228, 2005.

WALTHALL, W.K.; STARK, J.D. A comparison of acute mortality and population growth rate as endpoints of toxicological effect. Ecotoxicology and Environmental Safety, v.37, n.1, p.45-52, 1997.
ZILLI, J.E.; VALISHESKI, R.R.; FREIRE FILHO, F.R.; NEVES, M.C.P.; RUMJANEK, N.G. Assessment of cowpea rhizobium diversity in Cerrado areas of Northeastern Brazil. Brazilian Journal of Microbiology, v.35, n.4, p.281-287, 2004.

Recebido em 6/10/10

Aceito em 6/7/10 